\title{
On the Topological Sensitivity of Transient Acoustic Fields
}

\author{
Alison Malcolm \\ Department of Earth Sciences, Utrecht University \\ Now at: Department of Earth, Atmospheric and Planetary Sciences, \\ Massachussets Institute of Technology \\ Bojan Guzina \\ Department of Civil Engineering, University of Minnesota
}

\begin{abstract}
The concept of topological sensitivity has been successfully employed as an imaging tool to obtain the correct initial topology and preliminary geometry of hidden obstacles for a variety of inverse scattering problems. In this paper, we extend these ideas to acoustic scattering involving transient waveforms and penetrable obstacles. Through a boundary integral equation framework, we present a derivation of the topological sensitivity for the featured class of problems and illustrate numerically the utility of the proposed method for preliminary geometric reconstruction of penetrable obstacles. For generality, we also cast the topological sensitivity in the so-called adjoint field setting that is amenable to a generic computational treatment using, for example, finite element or finite difference methods.
\end{abstract}

\section{Introduction}

We consider the propagation of transient acoustic waveforms in the time domain and the inverse scattering problem of using recorded data to identify the support of penetrable obstacles where the acoustic medium parameters (velocity and density) differ from those in the background model. In the context of full waveform tomography, nonlinear minimization-based techniques for solving such inverse problems are intrinsically sensitive to the choice of the initial model. In particular, many such techniques get "trapped" in local minima of the featured cost functional when the initial model differs topologically from the true obstacle configuration. As shown in several recent studies, the topological derivative provides a preliminary point-probing functional for 
estimating the number, size, and location of obstacles from the recorded data and thus is a rational means to establish a topologically-correct initial "guess" for minimization purposes.

The idea behind the topological derivative is to probe each sampling point in the region of interest (suspected to contain the scatterers) and classify it as either part of the background or part of an inclusion, given the parameters of the background model and those of the inclusion(s). With reference to the cost function used as a basis for solving the inverse problem, this is done by introducing an infinitesimally small inclusion at a sampling point in the reference (i.e. background) medium and computing the derivative of the cost function with respect to such a perturbation. Because of the infinitesimal size of the trial inclusion, the resulting formula for the topological sensitivity, which relies on an asymptotic expansion of the scattered field for a vanishingly small obstacle, typically takes an explicit form and is inexpensive to compute.

The topological derivative was originally introduced in the doctoral thesis of Schumacher [1] as a computational tool for strucural shape optimization. Sokołowski and Żochowski [2-4] give the mathematical background underlying the technique and derive an explicit expression for the topological derivative in linear elasticity. Céa et al. [5] discuss the relationship between topological and shape optimization in the context of optimal structural design, and demonstrate the methods' utility through numerical examples. This work is continued in Garreau et al. [6] who examine the possibilities for combining shape and topological optimization. More recently, Novotny et al. [7] expose the relationship between the topological derivative and shape sensitivity analysis. To preserve the initial topology, they adapt the definition of the topological derivative so that an existing infinitesimal cavity is expanded, rather than introducing a new void. This work is continued in [8] where the topological sensitivity is compared with the domain truncation method under various boundary conditions prescribed on the boundary of an (infinitesimal) impenetrable obstacle. Lewiński and Sokołowski [9] give a comparison of several methods, including the topological derivative, for estimating the energy change of a system due to the introduction of an infinitesimal cavity.

Recently, Guzina and Bonnet [10] introduced the idea of using the topological derivative as a preliminary imaging tool in the context of inverse elastic scattering in the frequency domain. In [11] they introduce two alternative forms of the formula for the topological sensitivity and compare the effectiveness of the respective computational schemes. In $[12,13]$ the foregoing inverse scattering developments, limited to the reconstruction of impenetrable defects, are extended to deal with penetrable obstacles in acoustics [12] and elastodynamics [13], again involving time-harmonic waveforms.

To date the authors know of only two papers that discuss the topological 
derivative in the context of transient (acoustic or elastic) waveforms [14,15], although [16], which discusses sensitivity analysis for cracks in the time domain is certainly related. In the context of inverse scattering, performing this type of analysis in the time domain (as opposed to the frequency domain) is advantageous because in the time domain different phases, such as Rayleigh and compressional waves, can be separated and analyzed independently. Dominguez et al. [14] (see also [17]) derive time-domain formulas from the existing frequencydomain results and the Plancherel theorem. They demonstrate the relation between the topological derivative and time reversal methods for determining the source location [18-20], and discuss the utility of the former technique for non-destructive evaluation. Bonnet [15] gives a general elastodynamic framework for the topological derivative in the time domain, including a simplification to the acoustic case. Given the fact that both [14] and [15] deal with the nucleation of impenetrable obstacles (e.g. voids), the focus of this paper is a generalization in that it i) extends the application of the time-domain topological sensitivity to inverse scattering problems in acoustics involving penetrable obstacles (acoustic inclusions), and ii) employs the so-called direct formulation $[10,11]$ that has not been previously applied to transient problems.

The paper is organized as follows. The acoustic scattering problem and its boundary integral formulation are set up in sections 2 and 3, respectively, to permit acoustic wave scattering by homogeneous penetrable obstacles with arbitrary density and compressibility. The necessary framework for the topological derivative is established in the following two sections (4 and 5) which include the necessary asymptotics of the scattered field caused by a vanishing penetrable obstacle. The paper concludes with a set of 3D numerical examples relevant to the crosshole, non-linear waveform tomography [21-23] used in engineering geophysics.

\section{Preliminaries}

With reference to the active imaging configuration depicted in Fig. 1, our goal is to reconstruct homogeneous acoustic inclusions whose mass density and wave speed differ from those of the background medium. We define the reference (obstacle-free) medium $\Omega$ either as a homogeneous full-space or halfspace, with wave speed $c$ and mass density $\rho$. We next embed an inclusion $B_{\text {true }} \subset \Omega$ with boundary $S_{\text {true }}$, wave speed $c_{\text {true }}^{*}$, and mass density $\rho_{\text {true }}^{*}$. In this setting, the so-called background medium surrounding the obstacle is denoted $\Omega_{\text {true }}^{-}=\Omega \backslash B_{\text {true }}$. For inverse scattering purposes, we assume that the inclusion is illuminated with a transient source density, $E(\boldsymbol{\xi}, t)=g(\boldsymbol{\xi}) f(t)$ assumed to be continuous in $\Omega_{E} \times \mathbb{T}$, where $\Omega_{E} \subset \Omega$ is the (finite) support of $g$ assumed to be such that $\Omega_{E} \cap B_{\text {true }}=\emptyset$ and $\mathbb{T}=[0, T]$ is a closed time interval with $T$ being the maximum recorded time. We further assume that the 


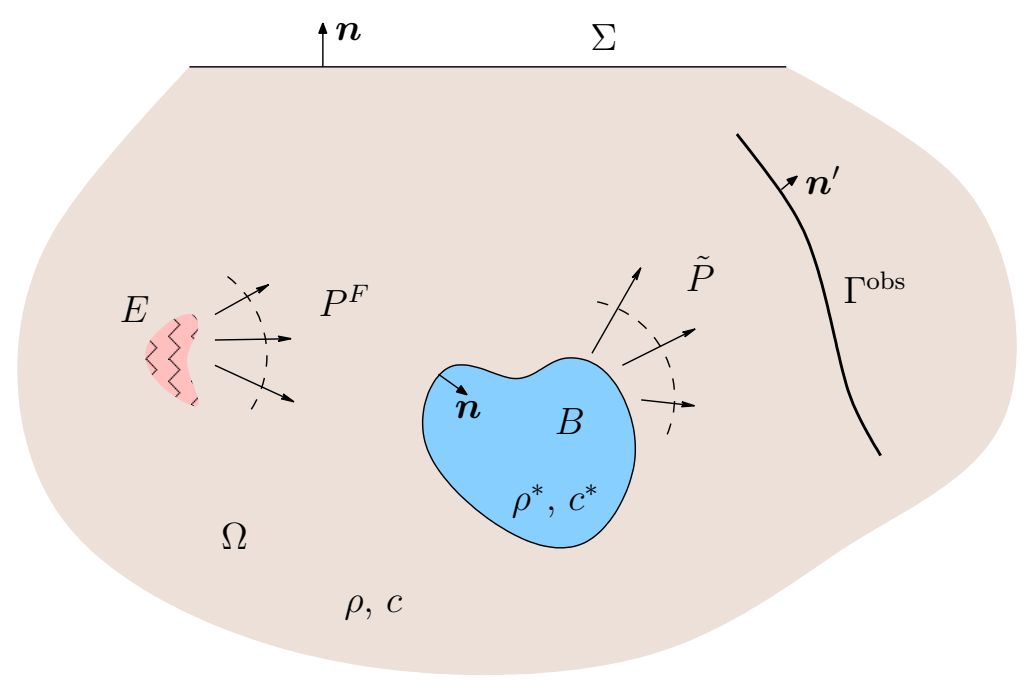

Fig. 1. Assumed geometry of the experiment. The goal is to determine the shape of $B_{\text {true. }}$

response of the system to this excitation (i.e. the acoustic pressure) is recorded over a measurement surface, $\Gamma^{\text {obs }} \subset \bar{\Omega}$. In what follows, this data set will be denoted by $P^{\text {obs }}$. In situations when $f(t)$ is given by a sequence of pulses, it is assumed that successive pulses do not interfere, i.e. that the recorded signal from one pulse has "died out" before the next one is set off. The forward problem then consists of solving for the pressure field, $P$ on $\Gamma^{\text {obs }} \times \mathbb{T}$ given the source function, the acoustic properties of both the background medium and the inclusion, and the geometry of the latter.

Our goal, on the other hand, is to solve the inverse problem of reconstructing the shape $B_{\text {true }}$ from knowledge of the data, $P^{\text {obs }}$, on $\Gamma^{\text {obs }} \times \mathbb{T}$, the background density, $\rho$, and velocity, $c$, and the material parameters of the obstacle $\left(\rho_{\text {true }}^{*}\right.$, $\left.c_{\text {true }}^{*}\right)$. A standard approach to this problem is to define a cost function that measures the distance between the observed and estimated data over the measurement surface; the preferred solution is then the one that minimizes this cost function. We select

$$
\mathcal{J}\left(\Omega^{-}, \beta, \gamma ; f\right)=\int_{0}^{T} d \tau \int_{\Gamma^{\mathrm{obs}}} \varphi\left(P, P^{\mathrm{obs}}, \boldsymbol{\xi}, \tau\right) d \Gamma_{\boldsymbol{\xi}},
$$

as the cost function, where $\gamma=c / c_{*} ; \beta=\rho / \rho_{*} ; P$ is an estimate of $P^{\text {obs }}$, computed for a trial obstacle $B$ with properties $c^{*}$ and $\rho^{*} ; \Omega^{-}=\Omega \backslash B$; and $\varphi$ is a real-valued non-negative (distance) function, differentiable with respect to its first argument. A commonly used example of $\varphi$ is

$$
\varphi\left(P, P^{\mathrm{obs}}, \boldsymbol{\xi}, \tau\right)=\frac{1}{2} W_{\boldsymbol{x}}(\boldsymbol{\xi}) W_{t}(\tau)\left|P(\boldsymbol{\xi}, \tau)-P^{\mathrm{obs}}(\boldsymbol{\xi}, \tau)\right|^{2}
$$

where $W_{x}$ and $W_{t}$ are respectively the spatial and temporal weighting functions, and $P^{\text {obs }}$ are the observed data $[14,15]$. 
With reference to (1), the topological derivative quantifies the sensitivity of $\mathcal{J}(\Omega)$ to the introduction of an infinitesimal inclusion in the reference (i.e. obstacle-free) domain $\Omega$ at a particular location. At those sampling points where the topological derivative attains negative values, the introduction of an infinitesimal defect by definition reduces the cost function; thus the properties of the "true" acoustic medium $\Omega_{\text {true }}^{-} \cup B^{\text {true }}$ (in terms of the wave speed and mass density) are expected to deviate from the background values at those locations. With reference to a simply-connected open set $\mathcal{B} \subset \mathbb{R}^{3}$ with boundary $\mathcal{S}$ we introduce the region $B_{\epsilon}=\boldsymbol{x}_{0}+\epsilon B \in \Omega$ (with volume $\epsilon^{3}|\mathcal{B}|$ ) as the support of the trial infinitesimal inclusion (assuming $\epsilon \rightarrow 0$ ) placed at the sampling point $\boldsymbol{x}_{0}$ in the reference medium. We assume the boundary of this region, $S_{\epsilon}=\partial B_{\epsilon}$, to be smooth of class $C^{1}$. Throughout this paper, it is assumed that the trial material parameters, $\left(c^{*}, \rho^{*}\right)$ of $B_{\epsilon}$ are equal to those of the "true" inclusion $\left(c_{\text {true }}^{*}, \rho_{\text {true }}^{*}\right)$.

We leave the reference shape $\mathcal{B}$ of the nucleated inclusion general, and define the topological derivative of the cost function $\mathcal{J}(\Omega)$, via the expansion

$$
\mathcal{J}\left(\Omega_{\epsilon}^{-}, \beta, \gamma ; E\right)=\mathcal{J}(\Omega, E)+\mathcal{T}\left(\boldsymbol{x}_{0}, \beta, \gamma ; E\right) h(\epsilon)+o(h(\epsilon)) \quad \text { as } \epsilon \rightarrow 0
$$

where $\Omega_{\epsilon}=\Omega \backslash B_{\epsilon}, \mathcal{J}(\Omega, E)$ denotes the value of $\mathcal{J}$ for the reference medium $(B=\emptyset)$, and $h(\epsilon)$ is to be determined. We assume that

$$
h(\epsilon)>0 \quad \lim _{\epsilon \rightarrow 0} h(\epsilon)=0, \quad \mathcal{T}\left(\boldsymbol{x}_{0}, \beta, \gamma ; E\right)<\infty
$$

for the topological derivative to be well-defined (see also [13]). The use of the term "derivative" for $\mathcal{T}$ is made by analogy with the Taylor series, noting that $\mathcal{T}$ plays the role of the first non-vanishing term in the Taylor expansion of $\mathcal{J}(\Omega)$. Although for the time being we assume the background medium to be homogeneous, the derivation easily generalizes to other cases in which the Green's function is available. In addition, in section 5.2 we derive an expression for the topological derivative in terms of the adjoint field; this formula does not require knowledge of the Green's function for the background medium.

To facilitate the ensuing developments, we assume that the acoustic pressure response $(P)$ of the system with a trial inclusion due to prescribed excitation $(E)$ can be divided into two parts, namely the free-field, $P^{F}$, defined as the response of the reference domain $\Omega$, and the scattered field, $\tilde{P}^{\epsilon}$, i.e. the perturbation component that vanishes as $\epsilon \rightarrow 0$. With reference to the latter observation, the key step in establishing the formula for $\mathcal{T}$ is an expansion of the featured cost functional $\mathcal{J}$ with respect to the scattered pressure field $\tilde{P}^{\epsilon}$ instead of $\epsilon$ in the limit as $\epsilon \rightarrow 0$.

To obtain the Taylor expansion of $\mathcal{J}(\Omega)$ with respect to $\tilde{P}^{\epsilon}$ in $(1)$ about the 
obstacle-free configuration $\left(\tilde{P}^{\epsilon}=0\right)$, we substitute the Taylor expansion

$$
\varphi\left(P, P^{\mathrm{obs}}, \cdot, \cdot\right)=\varphi\left(P^{F}, P^{\mathrm{obs}}, \cdot, \cdot \cdot\right)+\frac{\partial \varphi}{\partial P}\left(P^{F}, P^{\mathrm{obs}}, \cdot, \cdot\right) \tilde{P}^{\epsilon}+o\left(P^{\epsilon}\right) \text { as } \epsilon \rightarrow 0,
$$

of the cost function $\varphi$ into (1) resulting in

$$
\begin{aligned}
\mathcal{J}\left(\Omega_{\epsilon}^{-}, \beta, \gamma ; E\right)=\int_{0}^{T} d t & \int_{\Gamma^{\mathrm{obs}}}\left\{\varphi\left(P^{F}, P^{\mathrm{obs}}, \boldsymbol{\xi}, t\right)\right. \\
& \left.+\frac{\partial \varphi}{\partial P}\left(P^{F}, P^{\mathrm{obs}}, \boldsymbol{\xi}, t\right) \tilde{P}^{\epsilon}+o\left(\tilde{P}^{\epsilon}\right)\right\} d \Gamma_{\boldsymbol{\xi}} \text { as } \epsilon \rightarrow 0 .
\end{aligned}
$$

From (6) it is apparent that the topological sensitivity, as defined in (3), can be written as

$$
\mathcal{T}\left(\boldsymbol{x}_{0}, \beta, \gamma ; E\right)=\lim _{\epsilon \rightarrow 0} \frac{1}{h(\epsilon)} \int_{0}^{T} d t \int_{\Gamma^{\mathrm{obs}}} \frac{\partial \varphi}{\partial P}\left(P^{F}, P^{\mathrm{obs}}, \boldsymbol{\xi}, t\right) \tilde{P}^{\epsilon}(\boldsymbol{\xi}, t) d \Gamma_{\boldsymbol{\xi}},
$$

where $\frac{\partial \varphi}{\partial P}$ can be evaluated explicitly for any given $\varphi$, assumed to be differentiable with respect to its first argument. For example the derivative of the least-squares distance function (2) is

$$
\frac{\partial \varphi}{\partial P}\left(P^{F}, P^{\mathrm{obs}}, \boldsymbol{\xi}, t\right)=W_{\boldsymbol{x}}(\boldsymbol{\xi}) W_{t}(t)\left(P^{F}(\boldsymbol{\xi}, t)-P^{\mathrm{obs}}(\boldsymbol{\xi}, t)\right) .
$$

To evaluate $\mathcal{T}$ over a prescribed grid of sampling points $\boldsymbol{x}_{o}^{k}, k=1,2, \ldots K$, one would formally have to compute $\tilde{P}^{\epsilon}$ and thus solve the full $3 \mathrm{D}$ scattering problem $K$ times with the infinitesimal defect introduced separately at each sampling point $\boldsymbol{x}_{0}^{k}$. This is not practical, however, so in the sequel we derive an explicit asymptotic expression for $\tilde{P}^{\epsilon}$ as $\epsilon \rightarrow 0$.

\section{Boundary integral formulation of the forward scattering problem}

In this section we first state the forward problem as a system of partial differential equations. Following this, we derive the associated boundary integral equations to facilitate the asymptotic treatment necessary for the evaluation of the topological derivative (7).

To isolate the time dependence of the acoustic source $E(\boldsymbol{\xi}, t)=g(\boldsymbol{\xi}) f(t)$, we introduce the auxiliary (total) pressure field, $p$, via

$$
P(\boldsymbol{\xi}, t)=[f * p](\boldsymbol{\xi}, t), \quad(\boldsymbol{\xi}, t) \in\left(\Omega^{-} \cup B\right) \times \mathbb{T} .
$$

Here " $*$ " denotes the Riemann convolution, see (A.1); $P$ is the total pressure field in the inclusion-background system $\left(\Omega^{-} \cup B\right)$ due to the prescribed excitation $E$; and $p$ is the counterpart of $P$ when the excitation used to illuminate 
the obstacle is given by $E_{\delta}(\boldsymbol{\xi}, t)=g(\boldsymbol{\xi}) \delta(t)$ where $\delta$ denotes the Dirac delta function. By the linearity of convolution, we use a similar decomposition of this field into a free and scattered field as $p=p^{F}+\tilde{p}$. Additional properties of convolution used throughout the paper are given in Appendix A. In certain situations, $p^{F}$ can be estimated from $P^{F}$; this is the so-called deconvolution problem studied in many fields (see e.g. [24] for acoustic applications in the context of exploration seismology).

The free field $p^{F}$, generated by an impulsive source, $E_{\delta}(\boldsymbol{\xi}, t)=g(\boldsymbol{\xi}) \delta(t)$, satisfies the acoustic wave equation

$$
\nabla^{2} p^{F}(\boldsymbol{\xi}, t)-\frac{1}{c^{2}} \ddot{p}^{F}(\boldsymbol{\xi}, t)+\delta(t) g(\boldsymbol{\xi})=0, \quad \boldsymbol{\xi} \in \Omega, t \in \mathbb{T}
$$

subject to the Sommerfeld radiation condition at infinity. In situations when $\Omega$ is a semi-infinite domain bounded by the planar surface $\Sigma$ (located at $\xi_{3}=0$ ), the free field is further assumed to satisfy the boundary condition

$$
\alpha p^{F}+(1-\alpha) p_{, n}^{F}=0, \quad \boldsymbol{\xi} \in \Sigma,
$$

where $\alpha$ is either zero or one, i.e. $\alpha \in\{0,1\}$. In this context, $\alpha=0$ corresponds to Neumann boundary conditions and $\alpha=1$ to Dirichlet boundary conditions on $\Sigma$. We assume that all fields have a quiescent past, i.e.,

$$
p(\boldsymbol{\xi}, t)=\dot{p}(\boldsymbol{\xi}, t)=0, \quad \forall t<0, \boldsymbol{\xi} \in \Omega
$$

where $t=0$ is defined as the time when the source is first activated. By virtue of (12), the ensuing derivation and formulas apply to all $t \geq 0$.

With the foregoing definitions, the system of differential equations defining the forward scattering problem due to $E_{\delta}$ are

$$
\begin{aligned}
\nabla^{2} \tilde{p}-\frac{1}{c^{2}} \ddot{\tilde{p}}=0, & \boldsymbol{\xi} \in \Omega^{-}, \\
\nabla^{2} p-\frac{1}{c^{* 2}} \ddot{p}=0, & \boldsymbol{\xi} \in B, \\
\alpha \tilde{p}+(1-\alpha) \tilde{p}_{, n}=0, & \boldsymbol{\xi} \in \Sigma, \\
\tilde{p}+p^{F}=p, \quad \tilde{p}_{, n}+p_{, n}^{F}=\beta p_{, n}, & \boldsymbol{\xi} \in S,
\end{aligned}
$$

where $S=\partial B$ is the boundary of the trial obstacle, and "." indicates differentiation in time, see e.g. [25]. For clarity we note that the normal derivative of $p$ on $S$ with normal $\boldsymbol{n}$ (oriented toward the interior of $B$ ) in (16) is given by

$$
p_{, n}=\lim _{\gamma \rightarrow 0} \boldsymbol{n} \cdot \nabla p(\boldsymbol{\xi}+\gamma \boldsymbol{n}), \quad \boldsymbol{\xi} \in S,
$$

with analogous expressions applying for the normal derivatives of the component fields $p^{F}$ and $\tilde{p}$. As implied earlier, we require that all featured fields 
$\left(p, p^{F}\right.$ and $\left.\tilde{p}\right)$ satisfy the Sommerfeld radiation condition (written here in terms of the scattered field)

$$
\tilde{p}=O\left(\frac{1}{R}\right), \quad \tilde{p}_{, R}-\frac{1}{c} \dot{\tilde{p}}=o\left(\frac{1}{R}\right), \quad R \equiv|\boldsymbol{\xi}| \rightarrow \infty, \quad \boldsymbol{\xi} \in \Omega^{-}
$$

when $\Omega$ is a full-space, with analogous expressions (derived by the method of images) applying to the corresponding half-space problem. In particular for the latter problem, it is understood that $p, p^{F}$ and $\tilde{p}$ satisfy the respective radiation conditions as $|\boldsymbol{\xi}| \rightarrow \infty$ with $\boldsymbol{\xi}$ being restricted to $\xi_{3}>0$, where $\xi_{3}=0$ is the boundary of the half-space.

Following the development in [12], we next use Green's theorem to derive an integral representation

$$
\tilde{P}(\boldsymbol{x}, t)=\int_{S}\left\{\tilde{p}_{, n}(\boldsymbol{\xi}, t) * G(\boldsymbol{\xi}, \boldsymbol{x}, t)-\tilde{p}(\boldsymbol{\xi}, t) * H(\boldsymbol{\xi}, \boldsymbol{x}, t)\right\} d S_{\boldsymbol{\xi}}, \quad \boldsymbol{x} \in \Omega^{-},
$$

of the sought scattered field $\tilde{P}$ in terms of its time-impulsive counterpart $\tilde{p}$ over the boundary of the obstacle, $S$. Here $G$ and $H$ denote respectively the time-domain acoustic Green's function and its normal derivative due to the point source $\delta(\boldsymbol{\xi}-\boldsymbol{x}) f(t)$.

Substituting the boundary conditions on $S$ given in (16) for $\tilde{p}$ and $\tilde{p}_{, n}$, and applying Green's theorem for the second time yields the Somigliana-type integral representation

$$
\begin{aligned}
\tilde{P}(\boldsymbol{x}, t)=-\int_{B} \nabla \cdot\{ & \left(\beta \nabla p(\boldsymbol{\xi}, t)-\nabla p^{F}(\boldsymbol{\xi}, t)\right) * G(\boldsymbol{\xi}, \boldsymbol{x}, t) \\
& \left.-\left(p(\boldsymbol{\xi}, t)-p^{F}(\boldsymbol{\xi}, t)\right) * \nabla G(\boldsymbol{\xi}, \boldsymbol{x}, t)\right\} d V_{\boldsymbol{\xi}}, \quad \boldsymbol{x} \in \Omega^{-},
\end{aligned}
$$

where the "-" sign comes from the use of an inward-oriented normal $\boldsymbol{n}$. Note again that the Green's function used here contains the time-dependence of the source used to illuminate the obstacle. Expanding the divergence term and substituting (10) for $\nabla^{2} p^{F}$, (14) for $\nabla^{2} p$, and using the wave equation to replace $\nabla^{2} G$, results in the integral expression for the scattered field

$$
\begin{aligned}
\tilde{P}(\boldsymbol{x}, t)= & (1-\beta) \int_{B}\{\nabla p(\boldsymbol{\xi}, t) * \nabla G(\boldsymbol{\xi}, \boldsymbol{x}, t)\} d V_{\boldsymbol{\xi}} \\
& +\left(1-\beta \gamma^{2}\right) \int_{B}\left\{\frac{1}{c^{2}} p(\boldsymbol{\xi}, t) * \ddot{G}(\boldsymbol{\xi}, \boldsymbol{x}, t)\right\} d V_{\boldsymbol{\xi}}, \quad \boldsymbol{x} \in \Omega^{-} \backslash \Omega_{E},
\end{aligned}
$$

by virtue of the convolution property (A.4). Note that in arriving at equation (21) we assume that $\Omega_{E} \cap B=\emptyset$ which permits the cancellation of the source term in (10). 


\section{Acoustic field in the interior of a vanishing obstacle}

The idea of topological sensitivity $[10,11]$ is to introduce infinitesimally small obstacles (one at a time) into the reference medium, $\Omega$. To this end, we need an estimate of the scattered field, denoted by $\tilde{P}^{\epsilon}$, from the infinitesimal obstacle $B_{\epsilon}$ placed at a sampling point $\boldsymbol{x}_{0} \in \Omega$ in the limit as $\epsilon \rightarrow 0$. Because this obstacle is infinitesimally small, and the location of the observation point $\boldsymbol{x}$ is assumed to be at a fixed non-zero distance from the sampling point $\boldsymbol{x}_{0}$ inside the obstacle (resulting in $G(\boldsymbol{\xi}, \boldsymbol{x}, t-\tau)$ attaining only finite values), we replace $G$ with its zero-order Taylor expansion about $\boldsymbol{\xi}=\boldsymbol{x}_{0}$ which reduces (21) to

$$
\begin{aligned}
\tilde{P}^{\epsilon}(\boldsymbol{x}, t)= & (1-\beta)\left\{\int_{B_{\epsilon}} \nabla p^{\epsilon}(\boldsymbol{\xi}, t) d V_{\boldsymbol{\xi}}\right\} * \nabla G\left(\boldsymbol{x}, \boldsymbol{x}_{0}, t\right) \\
& +\left(1-\beta \gamma^{2}\right) \frac{1}{c^{2}}\left\{\int_{B_{\epsilon}} p^{\epsilon}(\boldsymbol{\xi}, t) d V_{\boldsymbol{\xi}}\right\} * \ddot{G}\left(\boldsymbol{x}, \boldsymbol{x}_{0}, t\right)+o\left(\epsilon^{3}\right) \\
& \epsilon \rightarrow 0, \quad \boldsymbol{x} \in \Omega^{-},
\end{aligned}
$$

which is the time-domain equivalent of formula (16) in [12].

To estimate the pressure field $p^{\epsilon}$ inside the scatterer, it is useful to formulate the acoustic transmission problem (13-16) in terms of a pair of boundary integral equations. We denote by $\kappa(\boldsymbol{x})$ the so-called free term [26], where $\kappa(\boldsymbol{x})=1 / 2$ if $S_{\epsilon}$ is smooth at $\boldsymbol{x}$. Accordingly, the pair of boundary integral equations describing (13-16) can be written as

$$
\begin{aligned}
& \kappa(\boldsymbol{x}) \tilde{P}(\boldsymbol{x}, t)=\kappa(\boldsymbol{x}) f(t) * \tilde{p}^{\epsilon}(\boldsymbol{x}, t) \\
& \quad=\int_{S_{\epsilon}}\left\{\tilde{p}_{, n}^{\epsilon}(\boldsymbol{\xi}) * G(\boldsymbol{\xi}, \boldsymbol{x}, t)-\tilde{p}^{\epsilon}(\boldsymbol{\xi}, t) * H(\boldsymbol{\xi}, \boldsymbol{x}, t)\right\} d S_{\boldsymbol{\xi}}, \quad \boldsymbol{x} \in S_{\epsilon},
\end{aligned}
$$

and

$$
\begin{array}{r}
\kappa(\boldsymbol{x}) f(t) * \tilde{p}^{\epsilon}(\boldsymbol{x}, t)+\frac{1}{\beta} \int_{S_{\epsilon}}\left\{\tilde{p}_{, n}^{\epsilon}(\boldsymbol{\xi}, t) * G^{*}(\boldsymbol{\xi}, \boldsymbol{x}, t)-\tilde{p}^{\epsilon}(\boldsymbol{\xi}, t) * H^{*}(\boldsymbol{\xi}, \boldsymbol{x}, t)\right\} d S_{\boldsymbol{\xi}}= \\
-\kappa(\boldsymbol{x}) f(t) * p^{F}(\boldsymbol{x}, t)-\frac{1}{\beta} \int_{S_{\epsilon}}\left\{p_{, n}^{F}(\boldsymbol{\xi}) * G^{*}(\boldsymbol{\xi}, \boldsymbol{x}, t)-p^{F}(\boldsymbol{\xi}, t) * H^{*}(\boldsymbol{\xi}, \boldsymbol{x}, t)\right\} d S_{\boldsymbol{\xi}}, \\
\boldsymbol{x} \in S_{\epsilon}
\end{array}
$$

where $G^{*}$ and $H^{*}$ are the counterparts of $G$ and $H$ for a medium with velocity $c^{*}$ and mass density $\rho^{*}$. We note that (23) and (24), which are written respectively with reference to $B_{\epsilon}$ and $\Omega_{\epsilon}^{-}$, are coupled through the interfacial conditions (16). From (24) we also see that the "forcing" term for these coupled equations comes from the boundary distribution of the free field, $p^{F}$ on the right-hand side of the equation.

From the above integral equations written in terms of $\tilde{p}^{\epsilon}$, we next derive an expansion of this quantity as $\epsilon \rightarrow 0$ in terms of the solution to the associated 
Laplace transmission problem. This is accomplished by noting that the singular part of the Green's function for the acoustic wave equation is given by the Green's function for the Laplace equation (see [26]). We first introduce an auxiliary field, $q$, about which $p^{\epsilon}$ is expanded for vanishing $\epsilon$ and then show, using (23) and (24), that this field is indeed the solution of the associated Laplace transmission problem.

To make explicit the dependence on $\epsilon$ (the vanishing obstacle size), we introduce the scaled variables

$$
\boldsymbol{z}=\frac{\boldsymbol{x}-\boldsymbol{x}_{0}}{\epsilon}, \quad \boldsymbol{\zeta}=\frac{\boldsymbol{\xi}-\boldsymbol{x}_{0}}{\epsilon}, \quad \boldsymbol{d}=\frac{\nabla p^{F}\left(\boldsymbol{x}_{0}, t\right)}{\left|\nabla p^{F}\left(\boldsymbol{x}_{0}, t\right)\right|}, \quad \boldsymbol{n}(\boldsymbol{\xi})=\boldsymbol{\eta}(\boldsymbol{\zeta}),
$$

where (as defined earlier) $\boldsymbol{x}_{0}$ is a point contained in $B_{\epsilon}$. It then follows from the asymptotic expansion of the Green's function (see e.g. [26]) that

$$
\begin{aligned}
G(\boldsymbol{\xi}, \boldsymbol{x}, t) & =\frac{1}{\epsilon} f(t) G(\boldsymbol{\zeta}, \boldsymbol{z})+O(1) \text { as } \epsilon \rightarrow 0, \\
H(\boldsymbol{\xi}, \boldsymbol{x}, t) & =\frac{1}{\epsilon^{2}} f(t) H(\boldsymbol{\zeta}, \boldsymbol{z})+O(1) \text { as } \epsilon \rightarrow 0 .
\end{aligned}
$$

On the basis of the scaling given in (25), the surface measure becomes

$$
d S_{\boldsymbol{\xi}}=\epsilon^{2} d S_{\boldsymbol{\zeta}}
$$

We next introduce the auxiliary fields, $\tilde{q}$ and $q^{F}$, so that

$$
\begin{aligned}
\tilde{p}^{\epsilon}(\boldsymbol{\xi}, t) & =\epsilon\left|\nabla p^{F}\left(\boldsymbol{x}_{0}, t\right)\right| \tilde{q}(\boldsymbol{\zeta})+o(\epsilon) \quad \text { as } \epsilon \rightarrow 0, \quad \boldsymbol{\xi} \in S_{\epsilon}, \\
p^{F}(\boldsymbol{\xi}, t) & =p^{F}\left(\boldsymbol{x}_{0}, t\right)+\nabla p^{F}\left(\boldsymbol{x}_{0}, t\right) \cdot\left(\boldsymbol{\xi}-\boldsymbol{x}_{0}\right)+o(\epsilon) \\
& \equiv q^{F}(t)+\epsilon\left|\nabla p^{F}\left(\boldsymbol{x}_{0}, t\right)\right| \boldsymbol{\zeta} \cdot \boldsymbol{d}+o(\epsilon) \quad \text { as } \epsilon \rightarrow 0, \quad \boldsymbol{\xi} \in S_{\epsilon} .
\end{aligned}
$$

Because $q^{F}(t)$ (i.e. the amplitude of the free field at $\boldsymbol{x}_{0}$ ) is independent of the spatial coordinates $\boldsymbol{\xi}$, it satisfies the Laplace equation for each time instant, and thus the boundary integral equation

$$
-\kappa(\boldsymbol{z}) q^{F}(t)-\frac{1}{\beta} \int_{\mathcal{S}} q_{, \eta}^{F}(t) G(\boldsymbol{\zeta}, \boldsymbol{z}) d S_{\boldsymbol{\zeta}}+\int_{\mathcal{S}} q^{F}(t) H(\boldsymbol{\zeta}, \boldsymbol{z}) d S_{\boldsymbol{\zeta}}=0, \quad \boldsymbol{z} \in \mathcal{S}
$$

where $\mathcal{S}=\left.S_{\epsilon}\right|_{\epsilon=1}$ as stated previously.

To better understand the $\tilde{q}$ field, we substitute (26)-(28) into (23), obtaining

$$
\begin{array}{r}
0=\left|\nabla p^{F}\left(\boldsymbol{x}_{0}, t\right)\right| * f(t)\left\{-\kappa(\boldsymbol{z}) \tilde{q}(\boldsymbol{z})+\int_{\mathcal{S}}\left\{\tilde{q}_{, \eta}(\boldsymbol{\zeta}) G(\boldsymbol{\zeta}, \boldsymbol{z})-\tilde{q}(\boldsymbol{\zeta}) H(\boldsymbol{\zeta}, \boldsymbol{z})\right\} d S_{\boldsymbol{\zeta}}\right\}+o(1) \\
\text { as } \quad \epsilon \rightarrow 0, \quad \boldsymbol{z} \in \mathcal{S}
\end{array}
$$


Equation (30) holds for every $t \geq 0$ if

$$
\begin{array}{r}
0=-\kappa(\boldsymbol{z}) \tilde{q}(\boldsymbol{z})+\int_{\mathcal{S}}\left\{\tilde{q}_{, \eta}(\boldsymbol{\zeta}, \tau) G(\boldsymbol{\zeta}, \boldsymbol{z})-\tilde{q}(\boldsymbol{\zeta}, \tau) H(\boldsymbol{\zeta}, \boldsymbol{z})\right\} d S_{\boldsymbol{\zeta}}+o(1) \\
\text { as } \epsilon \rightarrow 0, \quad \boldsymbol{z} \in \mathcal{S} .
\end{array}
$$

We now apply the same analysis to (24) employing (29) to obtain

$$
\begin{aligned}
\kappa(\boldsymbol{z}) \tilde{q}(\boldsymbol{z})+\frac{1}{\beta} \int_{\mathcal{S}} \tilde{q}_{, \eta}(\boldsymbol{\zeta}) G(\boldsymbol{\zeta}, \boldsymbol{z}) d S_{\boldsymbol{\zeta}}-\int_{\mathcal{S}} \tilde{q}(\boldsymbol{\zeta}) H(\boldsymbol{\zeta}, \boldsymbol{z}) d S_{\boldsymbol{\zeta}} \\
=-\kappa(\boldsymbol{z})(\boldsymbol{z} \cdot \boldsymbol{d})-\frac{1}{\beta} \int_{\mathcal{S}}(\boldsymbol{\eta} \cdot \boldsymbol{d}) G(\boldsymbol{\zeta}, \boldsymbol{z}) d S_{\boldsymbol{\zeta}}+\int_{\mathcal{S}}(\boldsymbol{\zeta} \cdot \boldsymbol{d}) H(\boldsymbol{\zeta}, \boldsymbol{z}) d S_{\boldsymbol{\zeta}}+o(1) \\
\text { as } \quad \epsilon \rightarrow 0, \quad \boldsymbol{x} \in \mathcal{S} .
\end{aligned}
$$

From the reduced integral equations (31)-(32) and the fact that $G$ is the Green's function for the Laplace equation, we find (cf. (23)-(24)) that $q$ must solve the Laplace transmission problem in $\mathbb{R}^{3}$, namely

$$
\begin{aligned}
\nabla_{\boldsymbol{\zeta}}^{2} \tilde{q}=0, & & \zeta \in \mathbb{R}^{3} \backslash \overline{\mathcal{B}} \\
\nabla_{\boldsymbol{\zeta}}^{2} q=0, & & \zeta \in \mathcal{B} \\
\tilde{q}+\boldsymbol{\zeta} \cdot \boldsymbol{d}=q, & & \zeta \in \mathcal{S} \\
\tilde{q}_{, \eta}+\boldsymbol{\eta} \cdot \boldsymbol{d}=\beta q_{, \eta}, & & \zeta \in \mathcal{S} .
\end{aligned}
$$

On the basis of (28) the total field, $p=p^{F}+\tilde{p}$, can now be expanded as

$$
\begin{aligned}
p^{\epsilon}(\boldsymbol{\xi}, t) & =p^{F}\left(\boldsymbol{x}_{0}, t\right)+\epsilon\left|\nabla p^{F}\left(\boldsymbol{x}_{0}, t\right)\right|(\boldsymbol{\zeta} \cdot \boldsymbol{d}+\tilde{q}(\boldsymbol{\zeta}))+o(\epsilon) \\
& =p^{F}\left(\boldsymbol{x}_{0}, t\right)+\epsilon\left|\nabla p^{F}\left(\boldsymbol{x}_{0}, t\right)\right| q(\boldsymbol{\zeta})+o(\epsilon) \quad \text { as } \epsilon \rightarrow 0, \boldsymbol{\xi} \in S_{\epsilon} .
\end{aligned}
$$

Because both $p^{\epsilon}(\boldsymbol{\xi})$ and $q(\boldsymbol{\zeta}(\boldsymbol{\xi}))$ are analytic (due to the fact that the source function $E(\boldsymbol{\xi}, t)$ is supported outside of $\left.B_{\epsilon}\right)$, the continuity statement (34), defined on $S_{\epsilon}=\partial B_{\epsilon}$, also holds in $B_{\epsilon}$ so that

$$
\begin{aligned}
p^{\epsilon}(\boldsymbol{\xi}, t) & =p^{F}\left(\boldsymbol{x}_{0}, t\right)+o(1), \\
\nabla p^{\epsilon}(\boldsymbol{\xi}, t) & =\left|\nabla p^{F}\left(\boldsymbol{x}_{0}, t\right)\right| \nabla_{\boldsymbol{\zeta}} q(\boldsymbol{\zeta})+o(1) \quad \text { as } \epsilon \rightarrow 0, \quad \boldsymbol{\xi} \in B_{\epsilon} .
\end{aligned}
$$

The relationship between $p^{\epsilon}$ and $p^{F}$ in (35) allows one to evaluate the second integral in (22) as

$$
\int_{B_{\epsilon}} p^{\epsilon}(\boldsymbol{\xi}, t) d V_{\boldsymbol{\xi}}=\epsilon^{3}|\mathcal{B}| p^{F}\left(\boldsymbol{x}_{0}, t\right)+o\left(\epsilon^{3}\right) \quad \text { as } \quad \epsilon \rightarrow 0
$$

where $\epsilon^{3}|\mathcal{B}|$ is the volume of $\mathcal{B}_{\epsilon}$. The evaluation of the first integral in (22), on the other hand, requires an expression for the $\nabla_{\zeta} q$ factor appearing in (35). 
Following [12] we find from (33) that $q$ is linear in $\boldsymbol{d}$ (as given by (25)) so that

$$
q(\boldsymbol{\zeta}) \equiv \boldsymbol{d} \cdot \boldsymbol{g}(\boldsymbol{\zeta}), \quad \nabla_{\boldsymbol{\zeta}} q(\boldsymbol{\zeta}) \equiv \boldsymbol{d} \cdot \nabla_{\boldsymbol{\zeta}} \boldsymbol{g}(\boldsymbol{\zeta}),
$$

where $\boldsymbol{g}$ satisfies the vector Laplace transmission problem

$$
\begin{array}{rlrl}
\nabla_{\zeta}^{2} \tilde{\boldsymbol{g}}=\mathbf{0}, & & \boldsymbol{\zeta} \in \mathbb{R}^{3} \backslash \mathcal{B} \\
\nabla_{\boldsymbol{\zeta}}^{2} \boldsymbol{g}=\mathbf{0}, & \boldsymbol{\zeta} \in \mathcal{B}, \\
\tilde{\boldsymbol{g}}+\boldsymbol{\zeta}=\boldsymbol{g}, & \boldsymbol{\zeta} \in \mathcal{S} \\
\tilde{\boldsymbol{g}}_{, \eta}+\boldsymbol{\eta}=\beta \boldsymbol{g}_{, \eta}, & \boldsymbol{\zeta} \in \mathcal{S} .
\end{array}
$$

By virtue of (25), (35) and (37), we arrive at the formula

$$
\int_{B_{\epsilon}} \nabla p^{\epsilon}(\boldsymbol{\xi}, t) d V_{\boldsymbol{\xi}}=\epsilon^{3}|\mathcal{B}| \nabla p^{F}\left(\boldsymbol{x}_{0}, t\right) \cdot \boldsymbol{A}+o\left(\epsilon^{3}\right) \quad \text { as } \epsilon \rightarrow 0,
$$

where

$$
\boldsymbol{A}=|\mathcal{B}|^{-1} \int_{\mathcal{B}} \nabla_{\zeta} \boldsymbol{g} d V_{\zeta}
$$

is a constant tensor, depending only on the shape of $\mathcal{B}$ chosen for the evaluation of $\mathcal{T}$ and the mass density contrast $(\beta)$ between the background and the inclusion. In situations when $\mathcal{B}$ is a unit ball, it can be shown from (38) and (40) that

$$
\boldsymbol{A}=\frac{3}{\beta+2} \boldsymbol{I}_{2}
$$

where $\boldsymbol{I}_{2}$ is the second-order identity tensor. Note that upon comparison with [12], $\boldsymbol{A}$ is the same as in the frequency domain case.

On substituting (36)-(40) into (22), we finally obtain

$$
\begin{aligned}
\tilde{P}^{\epsilon}(\boldsymbol{x}, t)=\epsilon^{3}|\mathcal{B}|\left\{( 1 - \beta ) \left(\nabla p^{F}\left(\boldsymbol{x}_{0}, t\right) \cdot \boldsymbol{A} * \nabla G\left(\boldsymbol{x}, \boldsymbol{x}_{0}, t\right)\right.\right. & \\
& \left.+\left(1-\beta \gamma^{2}\right) \frac{1}{c^{2}} \dot{p}^{F}\left(\boldsymbol{x}_{0}, t\right) * \dot{G}\left(\boldsymbol{x}, \boldsymbol{x}_{0}, t\right)\right\}, \quad \boldsymbol{x} \in \Omega^{-},
\end{aligned}
$$

which makes use of (A.4) and the extended definition of convolution (A.2) for vector functions.

\section{The Evaluation of Topological Sensitivity}

In this section we establish two alternative expressions for the topological sensitivity on the basis of (7). The first is a direct approach that relies on the knowledge of the Green's function for the background medium. The second approach makes use of the so-called adjoint field, which can be estimated numerically when the complexity of the reference model precludes the computation of the Green's function in closed form. 


\subsection{Direct Approach}

From (42) we directly evaluate (7), noting that

$$
h(\epsilon)=|\mathcal{B}| \epsilon^{3}
$$

to arrive at the expression

$$
\begin{aligned}
& \mathcal{T}\left(\boldsymbol{x}_{0}, \beta, \gamma ; E\right)=\int_{0}^{T} d t \int_{\Gamma^{\mathrm{obs}}} \frac{\partial \varphi}{\partial P}\left(P^{F}(\boldsymbol{\xi}, t), P^{\mathrm{obs}}(\boldsymbol{\xi}, t), \boldsymbol{\xi}, t\right) \\
& \quad\left\{(1-\beta)\left(\nabla p^{F}\left(\boldsymbol{x}_{0}, t\right) \cdot \boldsymbol{A} * \nabla G\left(\boldsymbol{\xi}, \boldsymbol{x}_{0}, t\right)+\left(1-\beta \gamma^{2}\right) \frac{1}{c^{2}} \dot{p}^{F}\left(\boldsymbol{x}_{0}, t\right) * \dot{G}\left(\boldsymbol{\xi}, \boldsymbol{x}_{0}, t\right)\right\} d \Gamma_{\boldsymbol{\xi}},\right.
\end{aligned}
$$

for the topological derivative in terms of the Green's function for the reference medium $\Omega$.

In the above expression, we have chosen to embed the prescribed source wavelet, $f(t)$, in the Green's function. To arrive at an expression that is more closely related to the experimental data, we can equivalently put this wavelet in the data, obtaining

$$
\begin{gathered}
\mathcal{T}\left(\boldsymbol{x}_{0}, \beta, \gamma ; E\right)=\int_{0}^{T} d t \int_{\Gamma^{\mathrm{obs}}} \frac{\partial \varphi}{\partial P}\left(P^{F}(\boldsymbol{\xi}, t), P^{\mathrm{obs}}(\boldsymbol{\xi}, t), \boldsymbol{\xi}, t\right) \\
\left\{(1-\beta)\left(\nabla P^{F}\left(\boldsymbol{x}_{0}, t\right) \cdot \boldsymbol{A}\right) * \nabla G_{\delta}\left(\boldsymbol{\xi}, \boldsymbol{x}_{0}, t\right)+\left(1-\beta \gamma^{2}\right) \frac{1}{c^{2}} \dot{P}^{F}\left(\boldsymbol{x}_{0}, t\right) * \dot{G}_{\delta}\left(\boldsymbol{\xi}, \boldsymbol{x}_{0}, t\right)\right\} d \Gamma_{\boldsymbol{\xi}},
\end{gathered}
$$

where $G_{\delta}$ is the Green's function for an impulsive time source.

From (45) we observe that the so-called "direct" formula for the topological sensitivity consists of a dipole term (in terms of $\nabla G$ ) and a monopole term (in terms of $G$ ), both dependent on the material (acoustic) properties of the inclusion. This is consistent with formula (23) of [12] for harmonic problems. In this case, however, we incorporate simultaneously the system response over the range of frequencies contained in the featured wavelet $f(t)$. We thus expect, as observed in [27], that we will obtain a higher quality reconstruction due to the use of what amounts to more data, i.e., the signal components at multiple frequencies.

\subsection{Adjoint Field Approach}

In most realistic domains, the Green's function $G$ is not easily determined. To deal with this problem, (44) can alternatively be written in terms of the socalled adjoint field, $P^{\star}$, which can be computed numerically from the recorded 
data. By analogy to the developments in $[12,26]$, the adjoint field can be shown to represent an acoustic solution for the reference, i.e defect-free domain subject to virtual excitation with the data residual $P^{F}-P^{\text {obs }}$ over the measurement surface $\Gamma^{o b s}$. Subject to the Sommerfeld radiation condition as applicable, this adjoint field solves the boundary value problem

$$
\begin{aligned}
\nabla^{2} P^{\star}(\boldsymbol{\xi}, t)-\frac{1}{c^{2}} \ddot{P}^{\star}(\boldsymbol{\xi}, t)=0, & \boldsymbol{\xi} \in \Omega \backslash \Gamma^{\mathrm{obs}} \\
{\left[\left[P^{\star}(\boldsymbol{\xi}, t)\right]\right]=0, } & \boldsymbol{\xi} \in \Gamma^{\mathrm{obs}} \\
{\left[\left[P_{, n^{\prime}}^{\star}(\boldsymbol{\xi}, t)\right]\right]=\frac{\partial \varphi}{\partial P}\left(P^{F}(\boldsymbol{\xi}, T-t), P^{\mathrm{obs}}(\boldsymbol{\xi}, T-t), \boldsymbol{\xi}, T-t\right), } & \boldsymbol{\xi} \in \Gamma^{\mathrm{obs}}(46) \\
\alpha P^{\star}(\boldsymbol{\xi}, t)+(1-\alpha) P_{, n}^{\star}(\boldsymbol{\xi}, t)=0, & \boldsymbol{\xi} \in \Sigma,
\end{aligned}
$$

where $\boldsymbol{n}^{\prime}$ is the unit normal on $\Gamma^{\text {obs }},[[g]]=\lim _{\tau \rightarrow 0} g\left(\boldsymbol{\xi}+\tau n^{\prime}\right)-g\left(\boldsymbol{\xi}-\tau n^{\prime}\right)$ and we have assumed that the measurements are not made on the boundary of the reference domain, i.e. that $\Gamma^{\mathrm{obs}} \cap \Sigma=\emptyset$.

On the basis of (46) where the reference medium (with homogeneous boundary conditions) is subjected to a virtual excitation in terms of the time-reversed data residual $\partial \varphi / \partial P\left(P^{F}, P^{\text {obs }}, \cdot, \cdot\right)$, we recognize

$$
\begin{aligned}
& P^{\star}(\boldsymbol{x}, t):= \\
& \int_{\Gamma^{\mathrm{obs}}} d \Gamma_{\boldsymbol{\xi}} \int_{0}^{T} d t^{\prime} \frac{\partial \varphi}{\partial P}\left(P^{F}\left(\boldsymbol{\xi}, T-t^{\prime}\right), P^{\mathrm{obs}}\left(\boldsymbol{\xi}, T-t^{\prime}\right), \boldsymbol{\xi}, T-t^{\prime}\right) G_{\delta}\left(\boldsymbol{\xi}, \boldsymbol{x}, t-t^{\prime}\right), \\
& \boldsymbol{x} \in \Omega \backslash \Gamma^{\mathrm{obs}}, \quad(47)
\end{aligned}
$$

as the solution for the adjoint field in terms of the time-impulsive Green's function, $G_{\delta}$, for the reference domain $\Omega$.

By means of (47), along with several changes of variables and the convolution identity (A.5), we find that (44) can be rewritten as

$$
\begin{aligned}
\mathcal{T}\left(\boldsymbol{x}_{0}, \beta, \gamma ; E\right)=(1-\beta)\left(\nabla P^{F}\right. & \left.\left(\boldsymbol{x}_{0}, t\right) \cdot \boldsymbol{A}\right)\left.* \nabla P^{\star}\left(\boldsymbol{x}_{0}, t\right)\right|_{t=T} \\
& +\left.\left(1-\beta \gamma^{2}\right) \frac{1}{c^{2}} \dot{P}^{F}\left(\boldsymbol{x}_{0}, t\right) * \dot{P}^{\star}\left(\boldsymbol{x}_{0}, t\right)\right|_{t=T} .
\end{aligned}
$$

While formally established for situations when the reference domain $\Omega$ is an acoustic full-space, the topological sensitivity formula (48) is general in the sense that it applies to arbitrary (bounded or unbounded) shapes $\Omega$; in this case the adjoint field $P^{*}$ satisfying (46) is computed numerically via a suitable (e.g. finite difference) solution technique. For completeness, we note that (48) can be reduced to the impenetrable obstacle case (see formula (52) in [15]) by setting $\beta=0$. 


\section{$6 \quad$ Numerical Examples}

In what follows, the utility of topological derivative (45) as a tool for preliminary acoustic-wave imaging of penetrable defects is illustrated through several examples involving transient excitation. For simplicity, the reference shape $\mathcal{B}$ of the nucleating inclusion is taken to be a ball of unit radius, whereby the second-order polarization tensor $\boldsymbol{A}$ featured in the "direct" formula (45) for the topological sensitivity (used in the ensuing calculations) is given by (41). We present two experimental configurations: a single penetrable obstacle $\left(\beta_{\text {true }}=2, \gamma_{\text {true }}=0.5\right)$ in an acoustic full-space with wave speed $c$ and mass density $\rho$ (Example 1$)$, and two penetrable obstacles $\left(\beta_{\text {true }}=1 / 3, \gamma_{\text {true }}=\right.$ 0.8 ) in the same reference medium (Example 2). The data are simulated using a time-harmonic boundary integral method [28] (specialized to acoustics) over the angular frequency range $\omega a / c=0.08-34$ with a step of 0.08 , where $a$ is the reference length. The frequency domain data were then multiplied by a wavelet in the frequency domain and Fourier-transformed to give the temporal signal. This approach avoids the domain discretization problems inherent to finite-difference or finite-element methods. Unless stated otherwise we use the raised cosine wavelet given, in the time domain, by

$$
R_{c}(t)=\operatorname{sinc}\left(f_{0} \bar{t}\right) \frac{\cos \left(\pi \alpha f_{0} \bar{t}\right)}{1-\left(2 \alpha f_{0} \bar{t}\right)^{2}}, \quad \bar{t}=\frac{c}{a}\left(t-t_{0}\right),
$$

where $t_{0}$ denotes temporal onset of excitation, and $\operatorname{sinc}(\mathrm{x})=\frac{\sin (\mathrm{x})}{\pi \mathrm{x}}$. In what follows, we use $\alpha=0.85$ and $c t_{0} / a=8$. Fig. 2 shows the location of sources and receivers for the examples considered, where $x=\xi_{1} / a, y=\xi_{2} / a$ and $z=\xi_{3} / a$. As examined earlier, the volume of interest is sequentially "illuminated" from each source point while monitoring the induced pressure $\left(P^{\text {obs }}\right)$ by all recievers simultaneousy. We have chosen a "deep cross-hole" configuration to maximize the recording aperture as suggested in [27].

For Example 1, the obstacle is a ball of radius $0.3 a$ centered at $(0.5 \mathrm{a}, 3 \mathrm{a}, 0.5 \mathrm{a})$. The inclusion characteristics, $\beta_{\text {true }}=2$ and $\gamma_{\text {true }}=0.5$, are chosen to mimic an obstacle with zero impedence contrast (defining the impedence as $\rho c$ ) as this is a case in which the obstacle would be difficult to image with traditional reflection based methods. Fig. 3 shows the $\mathcal{T}$-distribution for Example 1 (computed assuming $\beta=\beta_{\text {true }}$ and $\gamma=\gamma_{\text {true }}$ in (45)) in two sections passing through the centroid of the obstacle: one parallel to the testing grid, and the other orthogonal to it. As seen from the display, the shape of the inclusion is reasonably recovered.

In many applications such as seismology, however, it is not possible to excite and record at very low frequencies such as those inherently included in the raised cosine wavelet. To investigate whether or not these frequencies are necessary, we have also tested the topological derivative with the so-called Ricker 
(a)

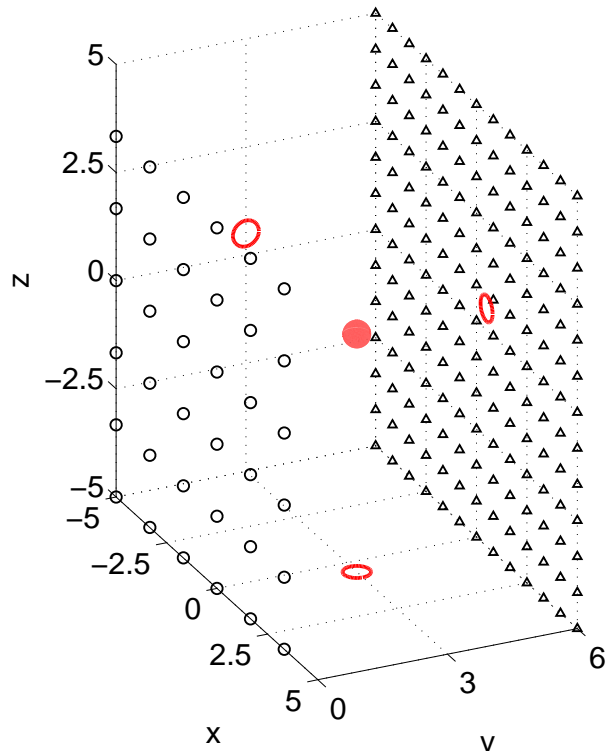

$\mathrm{X}$

y

(b)

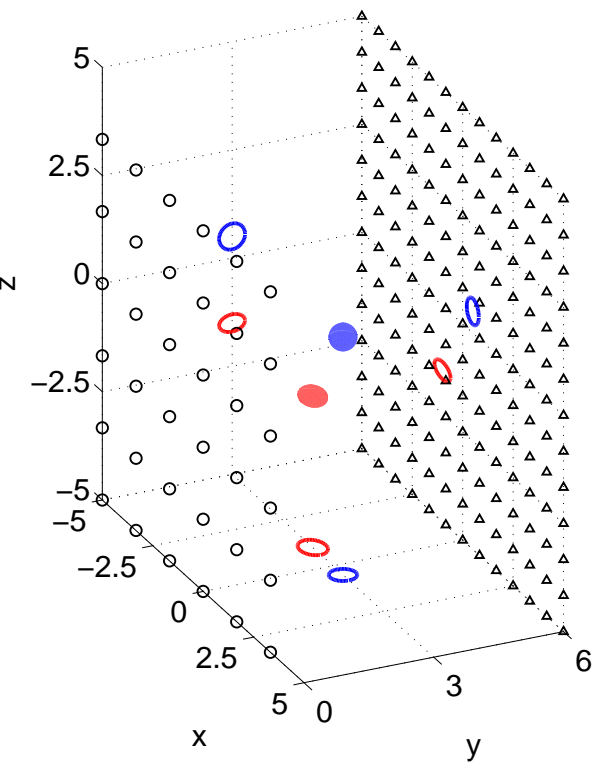

Fig. 2. Location of sources (circles) and receivers (triangles) used in the numerical examples: (a) Example 1 - single scatterer, and (b) Example 2 - two scatterers.

wavelet, given by

$$
R_{r}(t)=\left\{1-2\left(\frac{\pi f_{0} / \bar{t}}{2}\right)^{2}\right\} e^{-\left(\frac{\pi f_{0} \bar{t}}{2}\right)^{2}},
$$

which is commonly used in exploration seismology. From Fig. 4 we see that, while the two wavelets have dissimilar frequency contents, their use as a basis for acoustic obstacle reconstruction via the topological derivative results in relatively similar $\mathcal{T}$-distributions despite the fact that the Ricker wavelet does not contain a DC component. This is encouraging for possible applications to field data.

As indicated earlier, the results in Fig. 3 are obtained by setting $\beta=\beta_{\text {true }}$ and $\gamma=\gamma_{\text {true }}$ in (45). The results in Fig. 5 show, however, that a reasonable geometric reconstruction is possible even when the assumed parameters $\beta$ and $\gamma$ of the nucleating obstacle are incorrect. In the display, the distribution of topological derivative is shown when both $\beta$ and $\gamma$ are varied. The specific values are chosen so that the coefficients $(1-\beta)$ and $\left(1-\beta \gamma^{2}\right)$ of the two summands in (45) change sign. When the signs of both terms are correct, the object is identified through pronounced negative values of $\mathcal{T}$ as expected. If either sign is incorrect, on the other hand, the support of the obstacle correlates reasonably well with positive $\mathcal{T}$-values. This indicates that the topological derivative may also be useful in identifing whether the obstacle is "hard" or "soft"; this is discussed further in [13].

Fig. 6 illustrates the sectional reconstruction of the ellipsoidal defects for 

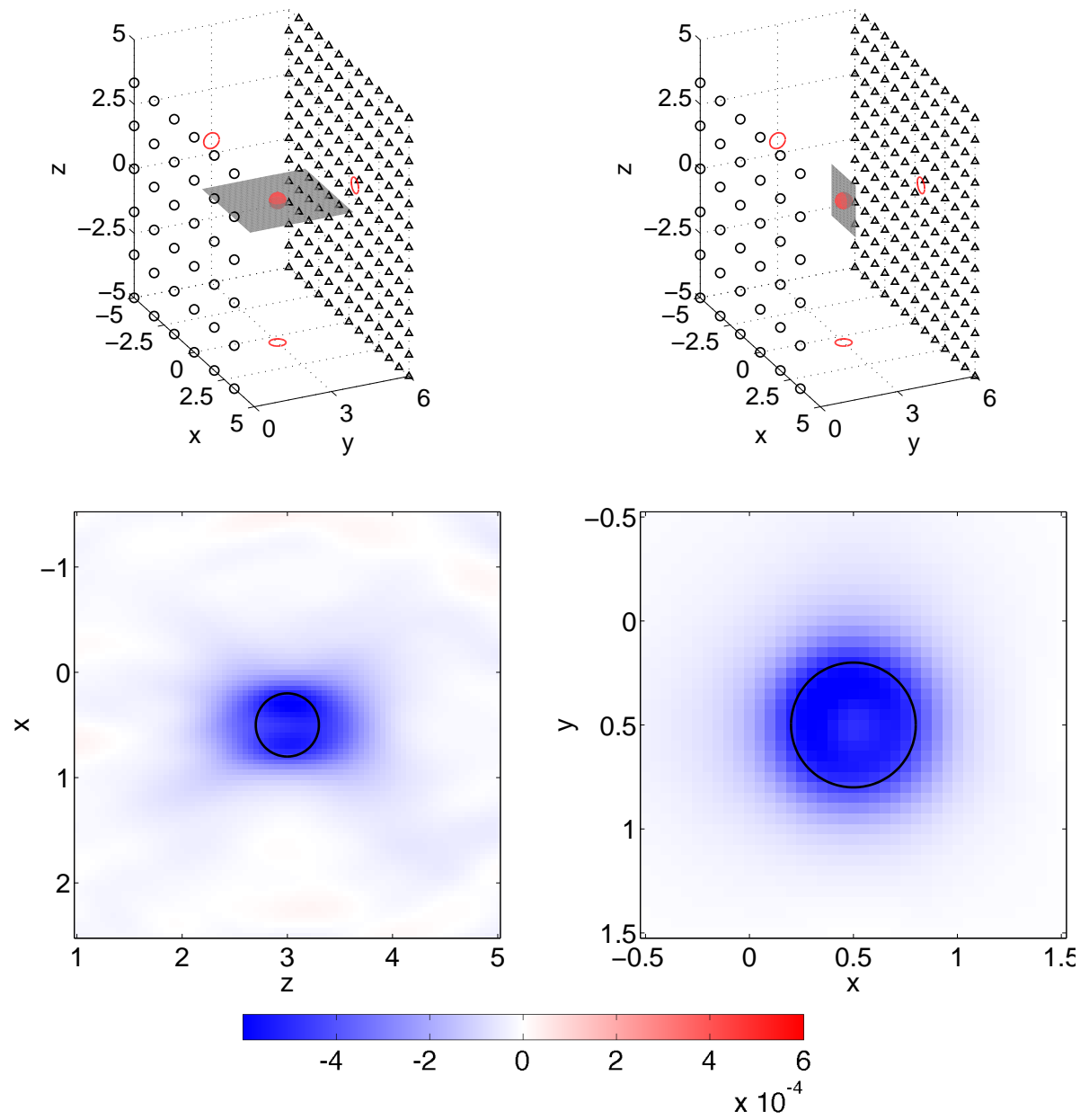

Fig. 3. Selected cross-sections (upper panels) and respective distributions of $\mathcal{T}$ (lower panels) for Example 1. Solid lines mark the intersection with the true obstacle. Both plots are made with central frequency $f_{0}=5$.

Example 2. The two obstacles, with principal semi-axes $(0.3 a, 0.3 a, 0.3 a)$ and $(0.4 a, 0.3 a, 0.2 a)$, are located respectively at $(0.5 a, 3 a, 0.5 a)$ and $(-a, 3 a,-1.5 a)$. In the example, both (penetrable) scatterers are characterized by $\beta_{\text {true }}=0.8$ and $\gamma_{\text {true }}=1 / 3$. These values were chosen as they signify the (upper) contrast limits in the Earth at seismic exploration scale, e.g. in the modeling of a salt inclusion in a sedimentary sequence. For generality, we compute the topological derivative with two different central frequencies, namely $f_{0}=3$ (left panels) and $f_{0}=5$ (right panels). In this case, we see that the reconstruction is arguably better at a lower frequency, most notably due to the absence of a "halo" effect characterizing the results for $f_{0}=5$. The latter effect, also observed in earlier time-harmonic studies $[12,13]$, can be best explained by the spatial oscillations embedded in the acoustic fields $P^{F}\left(\boldsymbol{x}_{0}, \cdot\right)$ and $G_{\delta}\left(\cdot, \boldsymbol{x}_{0}, \cdot\right)$ featured in (45). In general, one may expect that the wavelength of oscillations characterizing the spatial distribution of $\mathcal{T}$ would decrease with increasing $f_{0}$, whereby a pronounced negative "ridge" would be formed in the neighborhood 
(a)

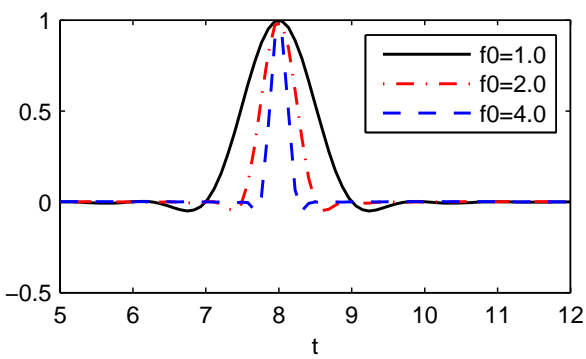

(b)

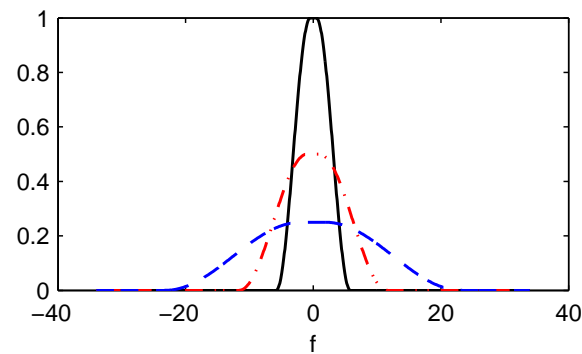

(c)

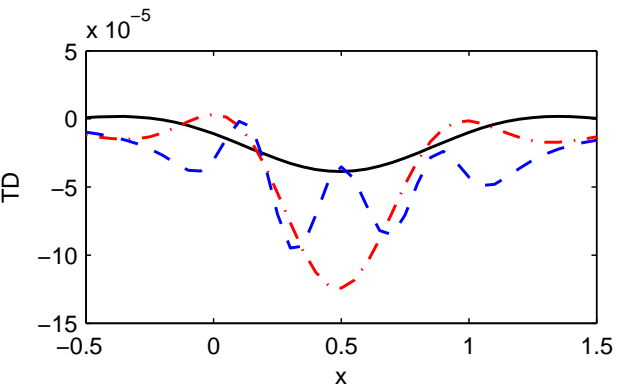

(d)

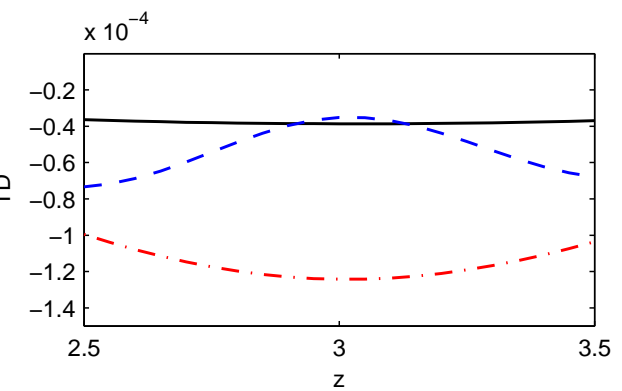

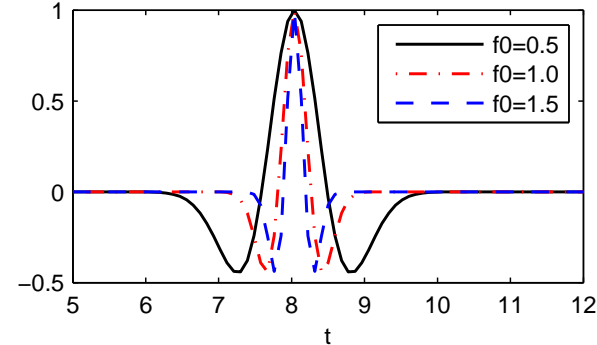
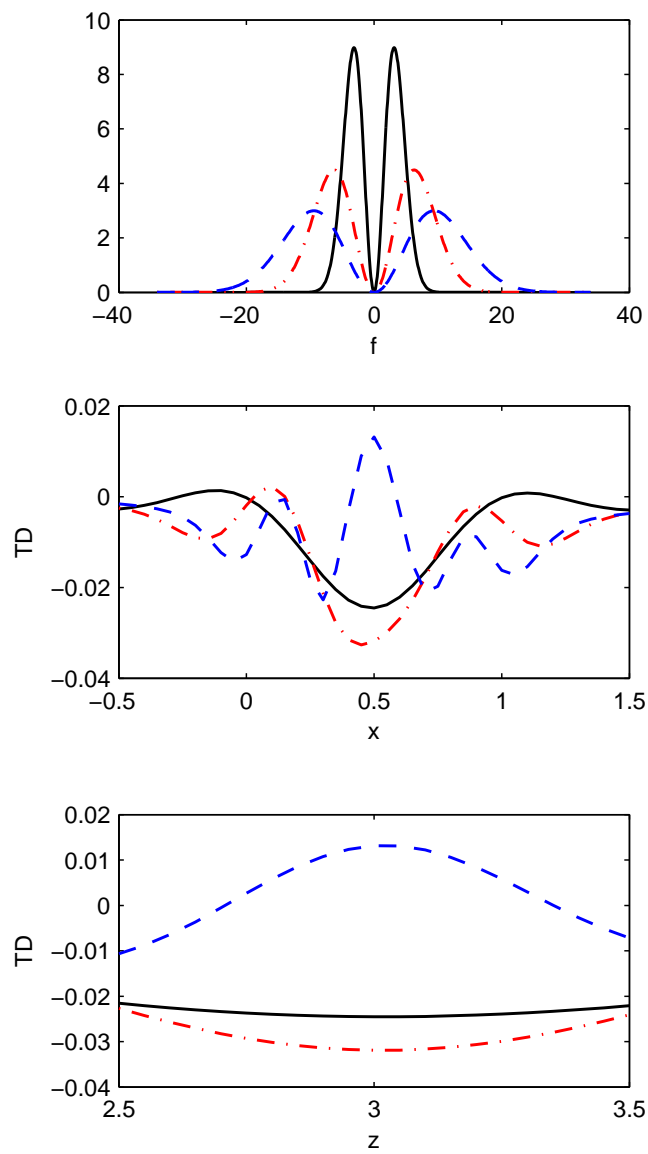

Fig. 4. Comparison between the raised cosine wavelet (left panels) and the Ricker wavelet (right panels). The central frequencies are: $f_{0}=1.0$ and 0.5 for the raised cosine and Ricker respectively (solid line), $f_{0}=2.0$ and 1.0 (dashed line), and $f_{0}=4.0$ and 2.0 (dashed-dot line). (a) is the wavelet in the time domain, (b) is the wavelet in the frequency domain, (c) is the distribution of the topological derivative for Example 1 along the line segment with $y=3.0$ and $z=0.5$, and (d) is the distribution of the topological derivative for Example 1 along the line segment with $x=0.5$ and $z=0.5$.

of the external surface of the obstacle(s) [13].

To demonstrate that there are no reconstruction artifacts in the space surrounding the two obstacles, Fig. 7 shows the full 3D reconstruction for Example 2. In the display, the volume between the sources and receivers $(-2.5<$ 


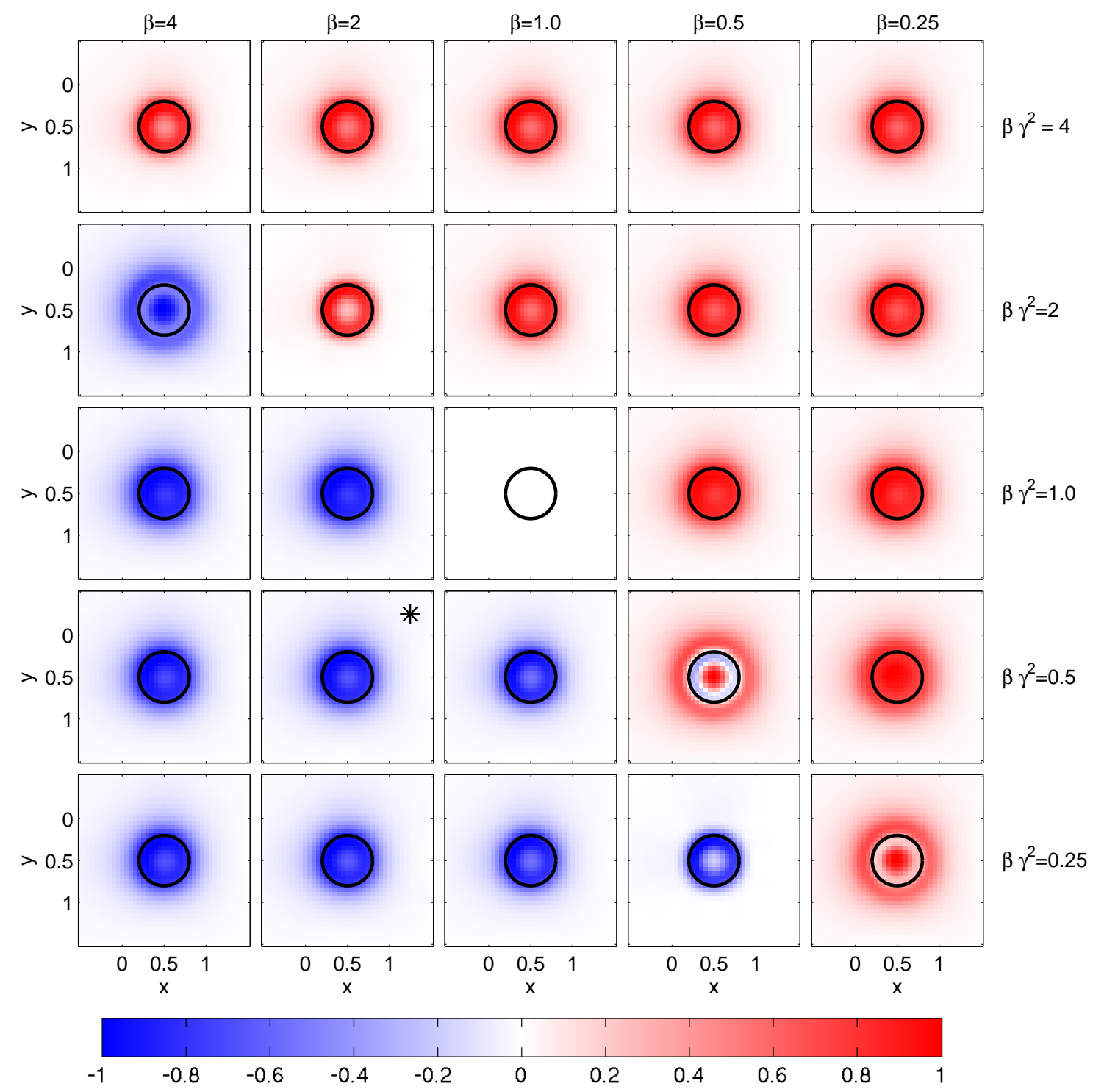

Fig. 5. Relative distribution of $\mathcal{T}$ in the $z=0$ plane for Example 1 under varying $\beta$ and $\gamma$. The star symbol indicates the distribution obtained with $\beta=\beta_{\text {true }}$ and $\gamma=\gamma_{\text {true }}$. All plots are generated with a raised-cosine wavelet with central frequency $f_{0}=5$.

$x<2,1<y<5$ and $-2.5<z<2$, see also Fig. 2b) is sampled through a uniform grid of $41 \times 46 \times 41$ points, and the scatterers are reconstructed using the $\mathcal{T}=0.45 \mathcal{T}^{\text {min }}$ isosurface where $\mathcal{T}^{\text {min }}$ is the extreme negative value of topological sensitivity computed over the sampled volume. 

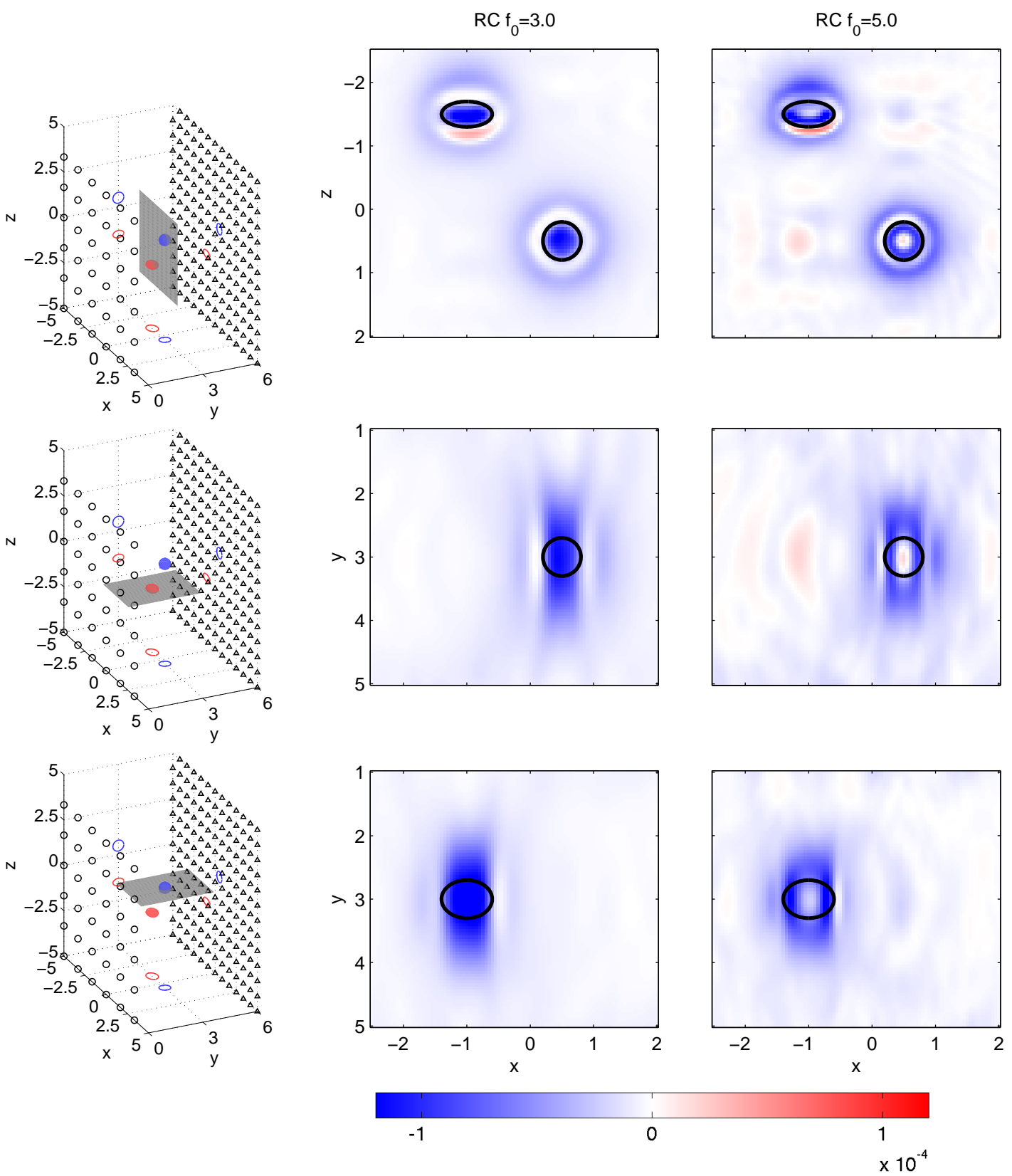

Fig. 6. Results for Example 2 (dual obstacle case). The diagrams on the left show the location of the selected cross-sections. The center panels show the $\mathcal{T}$-distribution computed with a raised cosine wavelet assuming $f_{0}=3$, while those on the right assume $f_{0}=5$. 


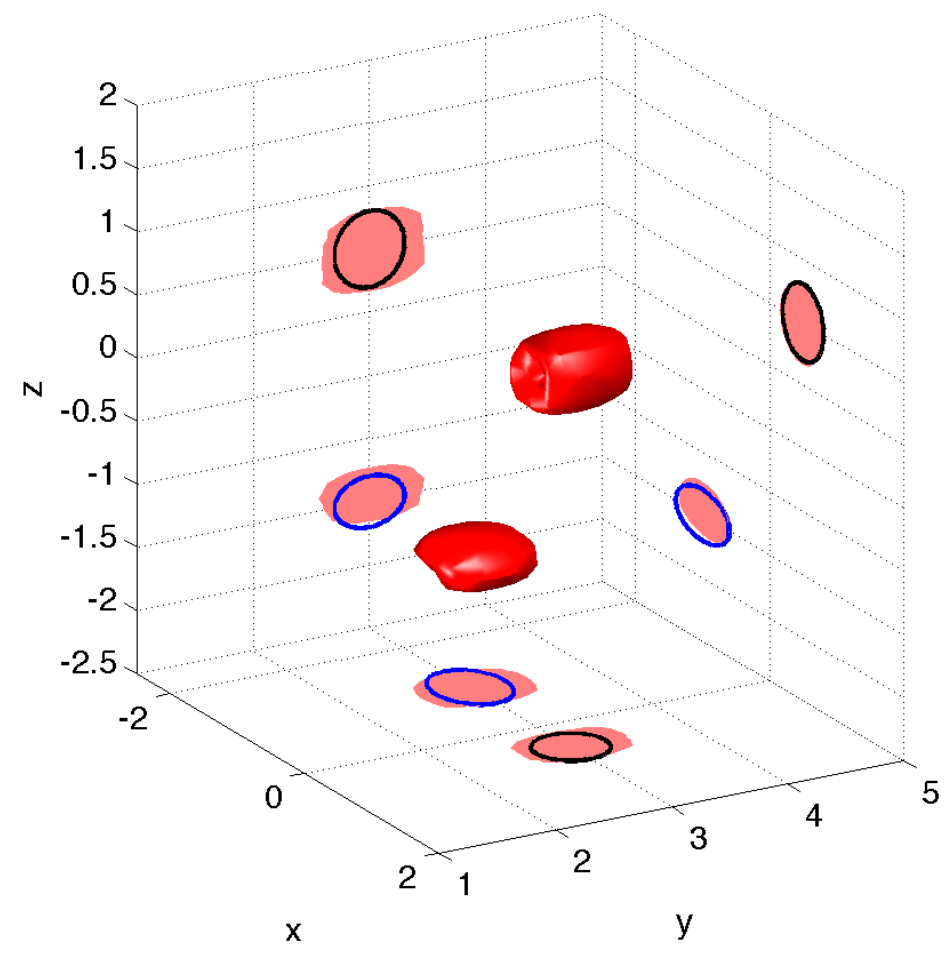

Fig. 7. 3D obstacle reconstruction for Example 2 (dual obstacle case). The two surfaces enclose the regions of the sampled space where the topological derivative takes negative values that are at least $45 \%$ of the extreme (negative) value. The shaded regions indicate projections onto coordinate planes where the true obstacle is outlined for the purpose of comparison. 


\section{Conclusions}

In this study, we extended the concept of the topological derivative to deal with inverse acoustic scattering in the time domain involving penetrable obstacles, giving expressions for the topological sensitivity both in terms of the Green's function and the adjoint field. These two techniques compliment each other, with the former being useful in cases where the background medium is well understood, and the latter when closed-form expressions for the Green's function are not readily available. Both variants of the formula for the topological sensitivity are derived using the so-called direct approach in which the key element is an asymptotic approximation of the scattered field due to an infinitesimal inclusion. This expansion is effected through an approximation of the acoustic field inside the vanishing obstacle by the solution of the associated Laplace transmission problem. With two simple numerical examples, we have shown that this concept allows for the preliminary recovery of the number, location, and size of penetrable obstacles from transient acoustic waveforms. We have further examined the influence of the wavelet shape and its central frequency. The extension to penetrable obstacles opens up the possibility of applying the topological derivative approach to problems in Earth sciences or medical imaging where the problem of interest is in identifying deviations from a known background medium.

\section{Acknowledgements}

A. Malcolm thanks the Institute for Mathematics and its Applications at the University of Minnesota where she was a postdoc during much of this work. A. Malcolm also acknowledges the support she received at Utrecht University through the Dutch National Science Foundation grant number NWO:VIVI865.03.007. The support provided by the National Science Foundation through Award No. CMS-0324348 to B. Guzina and the University of Minnesota Supercomputing Institute during the course of this investigation is kindly acknowledged. 


\section{A Some properties of convolution}

In this appendix we recall basic properties of convolution that are used throughout the paper. We define the causal or Riemann convolution [29] as

$$
[g * f](\boldsymbol{\xi}, t)= \begin{cases}\int_{0}^{t} f(\boldsymbol{\xi}, t-s) g(\boldsymbol{\xi}, s) d s, & t \geq 0 \\ 0, & t<0\end{cases}
$$

where $f$ and $g$ are any two scalar integrable functions of space and time. For vector functions, we define a convolution-like integral by

$$
[\boldsymbol{f} * \boldsymbol{g}]=\int_{0}^{t} \boldsymbol{f}(\boldsymbol{\xi}, t-s) \cdot \boldsymbol{g}(\boldsymbol{\xi}, s) d s
$$

where "." denotes the usual dot product. It follows directly from the above definitions that the following properties hold

$$
[f * g]=[g * f]
$$

and, assuming a quiescent past,

$$
\frac{\partial[f * g]}{\partial_{t}}=[\dot{f} * g]=[f * \dot{g}]
$$

as well as

$$
f(t) * g(T-t)=\int_{-\infty}^{\infty} f(t-\tau) g(T-\tau) d \tau
$$

where $T>0$ is a fixed time.

\section{References}

[1] A. Schumacher, Topologieoptimierung von bauteilstrukturen unter verwendung von lochpositionierungkriterien, Ph.D. thesis, Universität-GesamthochschuleSiegen, Siegen (1995).

[2] J. Sokołowski, A. Żochowski, On the topological derivative in shape optimization, SIAM J. Control Optim. 37 (1999) 1251-1272.

[3] J. Sokołowski, A. Żochowski, Topological derivatives for elliptic problems, Inverse Problems 15 (1999) 123-134.

[4] J. Sokołowski, A. Żochowski, Topological derivatives of shape functionals for elasticity systems, Mech. Struct. \& Mach. 29 (2001) 331-349. 
[5] J. Céa, S. Garreau, P. Guillaume, M. Masmoudi, The shape and topological optimizations connection, Comput. Methods Appl. Mech. Engrg. 188 (2000) 713-726.

[6] S. Garreau, P. Guillaume, M. Masmound, The topological asymptotic for PDE systems: The elasticity case, SIAM J. Control Optim. 39 (2001) 1756-1778.

[7] A. A. Novotny, R. A. Feijóo, E. Taroco, C. Padra, Topological sensitivity analysis, Comput. Methods Appl. Mech. Engrg. 192 (2003) 803-829.

[8] R. A. Feijóo, A. A. Novotny, E. Taroco, The topological derivative for the Poisson's problem, Mathematical Models and Methods in Applied Sciences 13 (2003) 1825-1844.

[9] T. Lewiński, J. Sokołowski, Energy change due to the appearance of cavities in elastic solids, International Journal of Solids and Structures 40 (2003) 17651803.

[10] B. B. Guzina, M. Bonnet, Topological derivative for the inverse scattering of elastic waves, Q. J. Mech. Appl. Math. 57 (2004) 161-179.

[11] M. Bonnet, B. B. Guzina, Sounding of finite solid bodies by way of topological derivative, Int. J. Numer. Meth. Engng. 61 (2004) 2344-2373.

[12] B. B. Guzina, M. Bonnet, Small-inclusion asymptotic for inverse problems in acoustics, Inverse Problems 22 (2006) 1761-85.

[13] B. B. Guzina, I. Chikichev, From imaging to material identification: A generalized concept of topological sensitivity, J. Mech. Phys. Solds 55 (2007) $254-79$.

[14] N. Dominguez, V. Gibiat, Y. Esquerre, Time domain topological gradient and time reversal analogy: An inverse method for ultrasonic target detection, Wave Motion 42 (2005) 31-52.

[15] M. Bonnet, Topological sensitivity for 3D elastodynamic and acoustic inverse scattering in the time domain, Comput. Methods Appl. Mech. Engrg 195 (2006) $5239-5254$.

[16] M. Bonnet, T. Burczyński, M. Nowakowski, Sensitivity analysis for shape pertubation of cavity or internal crack using BIE and adjoint variable approach, International Journal of Solids and Structures 39 (2002) 2365-2385.

[17] N. Dominguez, V. Gibiat, J. L. Arnaud, Flaw imaging with ultrasound: The time domain topological gradient method., AIP Conference Proceedings 760 (1) (2005) p859- 866 .

[18] M. Fink, C. Prada, F. Wu, D. Cassereau, Self focussing in inhomogeneous media with 'time reversal' acoustic mirrors, IEEE Ultrasonics Symposium (1989) 681686.

[19] M. Fink, Time reversal of ultrasonic fields - part I: Basic principles, IEEE Transactions on Ultrasonics, Ferroelectrics, and frequency control 39 (1992) $555-566$. 
[20] M. V. de Hoop, A. T. de Hoop, Wavefield reciprocity and optimization in remote sensing, Proc. R. Soc. Lond. A (Mathematical, Physical and Engineering Sciences) 456 (2000) 641-682.

[21] D. T. Reiter, W. Rodi, Nonlinear waveform tomography applied to crosshole seismic data, Geophysics 61 (1996) 902-913.

[22] P. V. Sharma, Environmental and Engineering Geophysics, Cambridge University Press, Cambridge, 1997.

[23] Y. Rao, Y. Wang, J. V. Morgan, Crosshole seismic waveform tomography - ii. resolution results, Geophys. J. Int. 166 (2006) 1237-1248.

[24] O. Yilmaz, Seismic data processing, Vol. 1 of Investigations in Geophysics No. 10, Society of Exploration Geophysicists, Tulsa, 2001.

[25] G. Dassios, R. Kleinman, Low Frequency Scattering, Clarendon Press, Oxford, 2000.

[26] M. Bonnet, Boundary integral equation methods for solids and fluids, John Wiley \& Sons, New York, 2006.

[27] I. Chikichev, Generalized topological sensitivity for inverse scattering of elastic waves, Ph.D. thesis, University of Minnesota (2007).

[28] R. Y. S. Pak, B. Guzina, Seismic soil-structure interaction analysis by direct boundary element methods, Int. J. Solids Struct. 36 (1999) 4743-4766.

[29] J. D. Achenbach, Wave propagation in elastic solids, North-Holland, Amsterdam, 1984. 